TITLE:

\title{
Toward solving the sign problem with path optimization method
}

$\operatorname{AUTHOR}(S)$ :

Mori, Yuto; Kashiwa, Kouji; Ohnishi, Akira

\section{CITATION:}

Mori, Yuto ... [et al]. Toward solving the sign problem with path optimization method. Physical Review D 2017, 96(11): 111501(R).

ISSUE DATE:

2017-12-01

URL:

http://hdl.handle.net/2433/233200

RIGHT:

(C) 2017 American Physical Society 
PHYSICAL REVIEW D 96, 111501(R) (2017)

\title{
Toward solving the sign problem with path optimization method
}

\author{
Yuto Mori, ${ }^{1, *}$ Kouji Kashiwa, ${ }^{2, \dagger}$ and Akira Ohnishi ${ }^{2, \$}$ \\ ${ }^{1}$ Department of Physics, Faculty of Science, Kyoto University, Kyoto 606-8502, Japan \\ ${ }^{2}$ Yukawa Institute for Theoretical Physics, Kyoto University, Kyoto 606-8502, Japan
}

(Received 17 September 2017; published 7 December 2017)

\begin{abstract}
We propose a new approach to circumvent the sign problem in which the integration path is optimized to control the sign problem. We give a trial function specifying the integration path in the complex plane and tune it to optimize the cost function which represents the seriousness of the sign problem. We call it the path optimization method. In this method, we do not need to solve the gradient flow required in the Lefschetzthimble method and then the construction of the integration-path contour arrives at the optimization problem where several efficient methods can be applied. In a simple model with a serious sign problem, the path optimization method is demonstrated to work well; the residual sign problem is resolved and precise results can be obtained even in the region where the global sign problem is serious.
\end{abstract}

DOI: 10.1103/PhysRevD.96.111501

\section{INTRODUCTION}

The sign problem induced by the oscillating Boltzmann weight of the partition function in the numerical integration for various quantum systems is serious obstruction in the computational science; see Ref. [1] for a review. Particularly, the sign problem attracts much more attention recently in QCD because some new approaches to circumvent the sign problem have been proposed and applied.

Recent promising approaches to evade the sign problem include the complex Langevin method [2-4] and the Lefschetz-thimble path-integral method [5-7]. The complex Langevin method is based on the stochastic quantization and then we are free from the complex weight. Therefore, the sign problem does not appear, but it is well known that the complex Langevin method sometimes provides us wrong results when the drift term shows singular behavior in the Langevin-time evolution [8]. In comparison, the Lefschetz-thimble path-integral method is based on the Picard-Lefschetz theory [9] and thus it is within the standard path-integral formulation. In this method, we construct the new integration-path contour which is so called the Lefschetz thimbles by solving the gradient flow starting from fixed points. Then, the partition function can be decomposed into the summation over contributions of relevant Lefschetz thimbles which can be determined from the crossing behavior of conjugate gradient flows with the original integration-path contour. On each Lefschetz thimble, the imaginary part of the action is constant and thus the sign problem seems to be resolved, but there are two remnants of the original sign problem. First one is the global sign problem: In the summation process of relevant Lefschetz thimbles, the cancellation can appear because imaginary parts of the action are constant on one Lefschetz thimble but have different values on

\footnotetext{
mori.yuto.47z@st.kyoto-u.ac.jp

kouji.kashiwa@yukawa.kyoto-u.ac.jp

¥ohnishi@yukawa.kyoto-u.ac.jp
}

different thimbles. The other is the residual sign problem; it comes from the Jacobian generated by the bending structure of the new integration path. Recently, one more serious problem in the Lefschetz-thimble path-integral method has been discussed which is so called the singularity problem: There are singular points and cuts on the complexified variables of integration if the action has the square root and/or the logarithm, explicitly and implicitly [10]. These singularities obstruct to draw continuous Lefschetzthimbles in the numerical calculation of gradient flows.

In this article, we propose a new method which we call the path optimization method to attack the sign and singularity problem. This method is motivated by the Lefschetz-thimble path-integral method. The main idea is the modification of the path-integral contour by minimizing the suitable cost function which reflects the seriousness of the sign problem. This means that the evading the sign problem arrives at the optimization problem. This fact becomes the strong advantage of this method because the optimization problem is well explored in the computational science and thus we may use several efficient methods such as the machine learning in the optimization process [11]. The path optimization method is demonstrated in the simple model with the serious sign problem where the complex Langevin method can fail.

\section{COST AND TRIAL FUNCTIONS}

In the path optimization method, the function which is so called the cost function plays a crucial role to construct the new integration-path contour on which the sign problem is controllable. The cost function is related with the seriousness of the sign problem with weakened weight cancellation by minimizing the function. In this article, we use the following cost function;

$$
\mathcal{F}[z(t)]=\frac{1}{2} \int d t\left|e^{i \theta(t)}-e^{i \theta_{0}}\right|^{2} \times\left|J(t) e^{-S(z(t))}\right|,
$$


with

$$
e^{i \theta(t)}=\frac{J(t) e^{-S(z(t))}}{\left|J(t) e^{-S(z(t))}\right|}, \quad e^{i \theta_{0}}=\frac{\mathcal{Z}}{|\mathcal{Z}|},
$$

where $z$ is the complexified variables of integration, $\mathcal{Z}$ is the partition function and $J(t)=d z / d t$. This function can be expressed by using the average phase factor as

$$
\frac{\mathcal{F}}{|\mathcal{Z}|}=\left|\left\langle e^{i \theta}\right\rangle_{p q}\right|^{-1}-1
$$

where

$$
\langle\mathcal{O}\rangle_{p q} \equiv \frac{\int d t \mathcal{O}\left|J e^{-S}\right|}{\int d t\left|J e^{-S}\right|}
$$

It should be noted that the choice of the cost function is not unique and thus we can freely change or extend it as long as the function reflects the seriousness of the sign problem.

To perform the optimization of Eq. (1), we need the trial function to specify the integration-path contour. One simple way to prepare the trial function is using the complete set, $\mathcal{H}_{m}$, as

$z(t)=x(t)+i y(t), \quad\left\{\begin{array}{l}x(t)=\sum_{m} c_{x, m} \mathcal{H}_{m}(t)+t, \\ y(t)=\sum_{m} c_{y, m} \mathcal{H}_{m}(t),\end{array}\right.$

with imposing the conditions, $x( \pm \infty)= \pm \infty,|y( \pm \infty)|$ $<\infty$. If the integrand, $\exp (-S)$, is analytic and suppressed rapidly enough with $|x| \rightarrow \infty$, the integrals on the original and modified paths are the same owing to Cauchy's integral theorem as long as the path does not go across singular points of $\exp (-S)$. It should be noted that we do not need to care the singular point of $S$ if it is not a singular point of $\exp (-S)$. We can extend this trial function to more complicated form by performing the feature engineering or the machine learning [11].

\section{EXAMPLE}

In this article, we consider the following partition function as an example to demonstrate the path optimization method. The actual form of partition function [8] is

$$
\mathcal{Z}_{p}=\int d x(x+i \alpha)^{p} e^{-\frac{x^{2}}{2}}
$$

where $\alpha$ and $p$ are input parameters and $p$ is a positive integer. The analytic result of $\mathcal{Z}_{p}$ can be obtained from the recurrence formula

$$
\mathcal{Z}_{p}=i \alpha \mathcal{Z}_{p-1}+(p-1) \mathcal{Z}_{p-2},
$$

and the expectation value of $x^{2}$ is expressed as

$$
\left\langle x^{2}\right\rangle_{p}=\frac{\mathcal{Z}_{p+2}-2 i \alpha \mathcal{Z}_{p+1}-\alpha^{2} \mathcal{Z}_{p}}{\mathcal{Z}_{p}} .
$$

In the path optimization method, we need to care the singular points of $\exp (-S)$. In the present action, the relevant singular points exist at $\operatorname{Im} z \rightarrow \pm \infty$. It should be noted that the singular point in $S$ at $z=-i \alpha$ does not cause any trouble. The factor of integrand $(z+i \alpha)^{p}$ leads to the action term of $-p \log (z+i \alpha)$ and causes the singular drift term, the drift term which diverges at $z=-i \alpha$, in the complex Langevin method [8]. However, $\exp (-S)$ is analytic at this point as long as $p$ is taken to be a positive integer, then it is not necessary to care in the path optimization method. Nevertheless, "singular point" indicates this zero point in the following discussions.

In the actual optimization, we use a simplified version of Eq. (5) based on the Gaussian function;

$$
\begin{gathered}
x(t)=t \\
y(t)=c_{1} \exp \left(-\frac{c_{2}^{2} t^{2}}{2}\right)+c_{3} .
\end{gathered}
$$

The optimization is numerically performed using the steepest descent method, $d c_{i} / d \tau=-\partial \mathcal{F} / \partial c_{i}$, and the integration is performed using the double exponential formula.

The optimized integration path in comparison with the Lefschetz thimble is shown in Fig. 1. It can be seen that the two contours overlap in the vicinity of the fixed point. However, there are qualitative differences on the thimble structure with varying $\alpha$. In the case with $\alpha=10$, Lefschetz thimbles terminate at the singular point unlike the case with $\alpha=15$ and then the optimized integration path approaches the singular point.

Figure 2 shows $J e^{-S}$ on the optimized integration path. We can see that there is the large probability distribution $\left(W(t) \sim\left|J e^{-S}\right|\right)$ with almost the constant phase near the fixed point. Therefore, Monte-Carlo sampling works with $\alpha=15$. In comparison, $W(t)$ has two peaks in the case with $\alpha=10$. The sign of $\operatorname{Im} J e^{-S}$ are opposite at both peaks and thus there are serious cancellations between them when we take into account both peaks to the integration. If we take into account only one peak, the wrong result comes up. The cancellations are induced by the singular point when the optimized integration path approaches to it: The Boltzmann weight becomes zero at the singular point and thus the sign of the Boltzmann weight can be easily flipped near the singular point. In the present case, there is exact parity symmetry between $\operatorname{Re} z$ and $-\operatorname{Re} z$ and thus the cancellation is very serious. This cancellation reflects the hidden Lefschetz thimble structure behind the path optimization method. 
TOWARD SOLVING THE SIGN PROBLEM WITH PATH ...
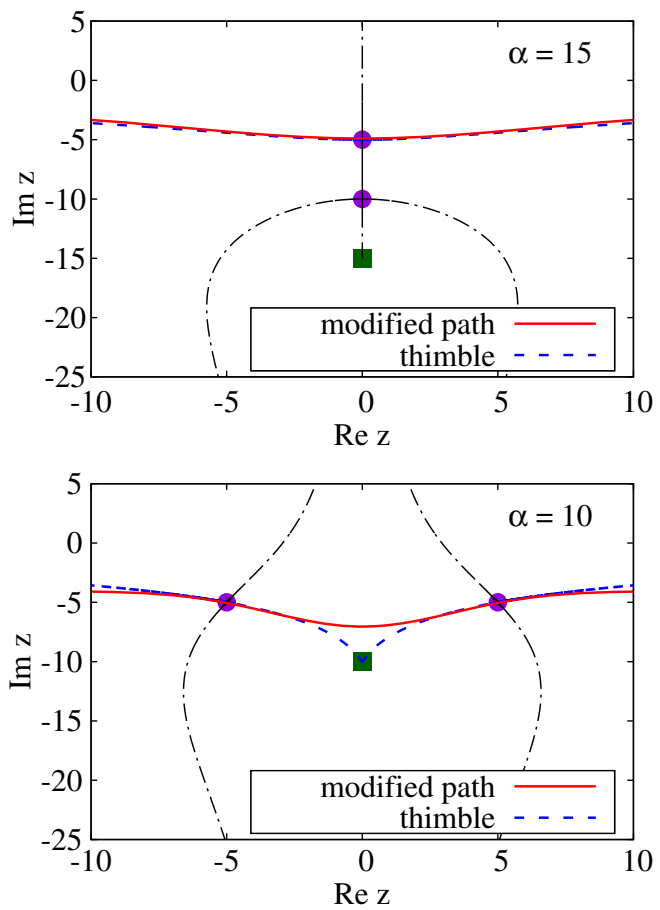

FIG. 1. Modified integration path by optimizing Eq. (1) and the Lefschetz thimbles for $p=50, \alpha=15,10$. Closed circle (square) point shows the fixed (singular) point. Dot-dashed lines are steepest ascent paths.
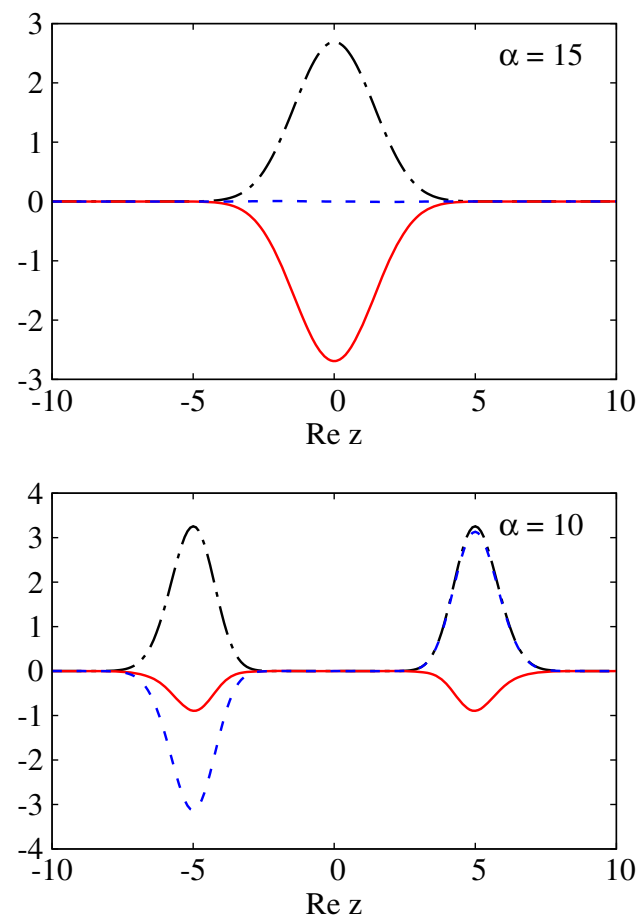

FIG. 2. Boltzmann weight on the modified integration path with $p=50, \alpha=15,10$. Solid (dashed) line represents real (imaginary) part of $J e^{-S}$. The dot-dashed line indicates $\left|J e^{-S}\right|$. Where, the amplitudes is normalized by factor $10^{55}$ with $\alpha=15$, and $10^{42}$ with $\alpha=10$.
PHYSICAL REVIEW D 96, 111501(R) (2017)

The optimized average phase factor is shown in Fig. 3. From the difference between the full calculation and the calculation in the $\operatorname{Re} z \in[0, \infty)$ range, we can clarify the seriousness of the global sign problem. In the case with $\alpha \gtrsim 14$, we can see that the sign problem can be solved because the path is represented by one thimble. In the case with $\alpha \lesssim 14$, contributions from the two thimbles cancel with each other. In the path optimization method, we can resolve the residual sign problem, but not the global sign problem. This problem also exists in the ordinary and generalized Lefschetz thimble methods [12].

Figure 4 shows the expectation value of $x^{2}$ in the hybrid Monte-Carlo method on the modified integration path. We calculate the expectation value in Eq. (4) by using the reflection symmetry of the Boltzmann weight, $W(t)=$ $W(-t)$, in this setting. This treatment replicates the parallel tempering algorithm which has been applied to the generalized Lefschetz-thimble path-integral method $[13,14]$. The results well agree with the analytic results (8). Readers can find the calculation of this model by using the complex Langevin method in Ref. [8].

Because the path optimization method is a variational method, we can restrict the variational space to represent integration path. This will be useful for multiple integral

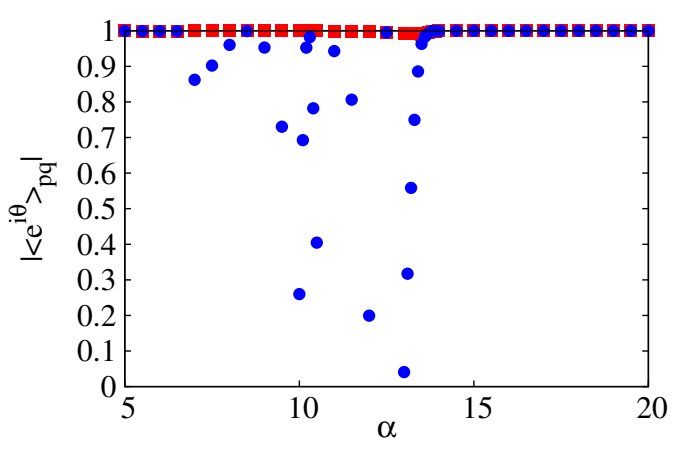

FIG. 3. The average phase factor with $p=50$. Closed circle shows the expectation in $\operatorname{Re} z \in(-\infty, \infty)$, and square point in $\operatorname{Re} z \in[0, \infty)$. The expectation value is calculated by numerical integration.

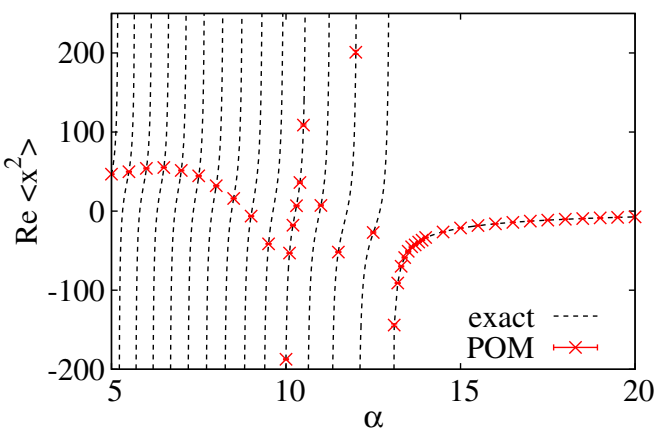

FIG. 4. The expectation value of $x^{2}$ with $p=50$. Errors are estimated by Jackknife method. 
problems such as quantum field theory. In addition, the path optimization method has large extensibility because we can freely replace or extend the cost function as long as it reflects the seriousness of the sign problem. These points are important advantages of the path optimization method. We leave the actual extension of the trial function and the cost function to a future work since this study is the first attempt to demonstrate our method.

\section{SUMMARY}

In this article, we have proposed a new approach to circumvent the sign problem which is motivated by the Lefschetz-thimble path-integral method. We call it the path optimization method. In the method, the new integration path is constructed in the plane of complexified variables of integration by minimizing the cost function. The cost function is set to reflect the seriousness of the sign problem. The actual optimization of the integration path is carried out by using the trial function.

We have demonstrated the path optimization method by using the simple model with the serious sign problem proposed in Ref. [8]. In the path optimization method, we can resolve the residual sign problem which appears in the ordinary and generalized Lefschetz-thimble path-integral methods. But, at least on our present choice of the cost function and in the present setting, the global sign problem cannot be resolved. However, we can well reproduce the exact results by using the path optimization method in the wide range of the model parameter space.

Finally, we summarize advantages of the path optimization method:

(1) No residual sign problem.

(2) Applicability of various efficient methods to the optimization process.

(3) Controllability of the singularity problem.

(4) Large extensibility of the cost function.

Possible disadvantage may be the numerical cost. In the complex system, the sign-problem weakened integration path is expected to have a very complicated shape. Therefore, we should check which optimization method is suitable or not step by step in the future.

\section{ACKNOWLEDGMENTS}

A. O. is supported in part by the Grants-in-Aid for Scientific Research from JSPS (Nos. 15K05079, 15H03663, 16K05350), the Grants-in-Aid for Scientific Research on Innovative Areas from MEXT (Nos. 24105001, 24105008), and by the Yukawa International Program for Quark-hadron Sciences (YIPQS).
[1] P. de Forcrand, Proc. Sci., LAT2009 (2009) 010, [arXiv: 1005.0539].

[2] G. Parisi and Y.-S. Wu, Sci. Sin. 24, 483 (1981).

[3] G. Parisi, Phys. Lett. 131B, 393 (1983).

[4] G. Aarts, E. Seiler, and I.-O. Stamatescu, Phys. Rev. D 81, 054508 (2010).

[5] E. Witten, AMS/IP Stud. Adv. Math. 50, 347 (2011).

[6] M. Cristoforetti, F. Di Renzo, and L. Scorzato (Aurora Science Collaboration), Phys. Rev. D 86, 074506 (2012).

[7] H. Fujii, D. Honda, M. Kato, Y. Kikukawa, S. Komatsu, and T. Sano, J. High Energy Phys. 10 (2013) 147.
[8] J. Nishimura and S. Shimasaki, Phys. Rev. D 92, 011501 (2015).

[9] F. Pham, in Proc. Symp. Pure Math (AMS, Providence, 1983), p. 319.

[10] Y. Mori, K. Kashiwa, and A. Ohnishi, arXiv:1705.03646.

[11] Y. Mori, K. Kashiwa, and A. Ohnishi, arXiv:1709.03208.

[12] A. Alexandru, G. Basar, and P. Bedaque, Phys. Rev. D 93, 014504 (2016).

[13] M. Fukuma and N. Umeda, Prog. Theor. Exp. Phys. 2017, 073B01 (2017).

[14] A. Alexandru, G. Basar, P. F. Bedaque, and N. C. Warrington, Phys. Rev. D 96, 034513 (2017). 